\title{
Challenges for Inclusive Affective Detection in Educational Scenarios
}

\author{
Olga C. Santos ${ }^{1}$, Alejandro Rodriguez-Ascaso ${ }^{1}$, Jesus G. Boticario ${ }^{1}$, \\ Sergio Salmeron-Majadas ${ }^{1}$, Pilar Quirós ${ }^{2}$, and Raúl Cabestrero ${ }^{2}$ \\ ${ }^{1}$ aDeNu Research Group. Artificial Intelligence Dept. Computer Science School. UNED \\ C/Juan del Rosal, 16. Madrid 28040, Spain \\ ${ }^{2}$ Basic Psychology Dept. UNED.C/Juan del Rosal, 10. Madrid 28040, Spain \\ \{ocsantos, arascaso, jgb\} @dia.uned.es, ssalmeron@bec.uned.es, \\ \{pquiros, rcabestrero\} apsi.uned.es
}

\begin{abstract}
There exist diverse challenges for inclusive emotions detection in educational scenarios. In order to gain some insight about the difficulties and limitations of them, we have analyzed requirements, accommodations and tasks that need to be adapted for an experiment where people with different functional profiles have taken part. Adaptations took into consideration logistics, tasks involved and user interaction techniques. The main aim was to verify to what extent the same approach, measurements and technological infrastructure already used in previous experiments were adequate for inducing emotions elicited from the execution of the experiment tasks. In the paper, we discuss the experiment arrangements needed to cope with people with different functional profiles, which include adaptations on the analysis and results. Such analysis was validated in a pilot experiment with 3 visually impaired participants.
\end{abstract}

\section{Introduction}

Our current research framed in the MAMIPEC project (TIN2011-29221-C03-01) focuses on exploring the application of affective computing to develop accessible and personalized learning systems that consider a user context where appliances and devices are used to provide a richer and more sensitive user interaction [1]. To detect emotions from users' interactions in an e-learning environment, we designed an inclusive individual large-scale experiment aimed to record multiple measures (qualitative, self-reported, physiological and behavioral). The participation of users with disabilities in such experiment posed several challenges. For example, the logistics of the whole experiment was considered, including the accessibility of the informed consent or other relevant questionnaires that had to be filled out by the users. Also, the accessibility and usability aspects of the interaction with the hardware and software were taken into account (e.g. replacement of visual stimuli by some other ways of access - such as audio recordings - in visually impaired users), both with traditional and emerging user-computer interaction techniques [2]. Furthermore, applicability of computer-based affect detection techniques to users with different abilities deserved specific analysis (e.g. detection of keyboard related events instead of mouse pressure for those who cannot use the mouse). In this context, we analyzed the requirements to 
gather emotional data from people with different abilities, including people with disabilities. Such analysis has been validated in a pilot with 3 visually impaired participants. Background for the work and experiments carried out are presented next.

\section{$2 \quad$ Related Works}

According to existing literature, learners' affective state features a strong relationship with the cognitive process [3]. Affective modeling is a sub-area of affective computing [4] that involves i) detection of users' emotion and ii) adaptation of the system response to the users' emotional state. User experience (UX) is a related HCI area, which is also relevant to our research activities. UX involves two important qualities: traditional HCI usability and accessibility balanced with hedonic and affective design. The latter improve user personal (as well as others') situation in relation with the elearning resource, as they will not only guide the future behaviour of the individual, but also because these subjective aspects will be communicated to others [5], and therefore will have a crucial effect on how the learning system is to be adopted by professionals and students in real situations.

Affect detection follows different approaches: i) qualitative methods (i.e. interviews, think-aloud [6]), ii) self-reported information, which can be gathered through questionnaires, iii) behavioral information can be obtained from either interactions with access devices, such as mouse and keyboards [7], or by using behavioral observational scales, where the participant behavior is coded on pre-assigned categories or by means of hardware sensor data that collect facial expressions, posture analysis, pressure on the mouse, etc. [8], and iv) physiological or neurological information (i.e. Electrocardiogram -ECG-, Galvanic Skin Response-GSR-, functional magnetic resonance imaging -fMRI-, etc.). Most of the technology and tools involved in detecting affective states in the educational domain are common to other affective computing areas [9]. Combinations of multiple sources of data and contextual information have improved the performance of affect recognition [10].

According to [11] user experience measurement should essentially be self-reported, trajectory-based and adaptive. They state that traditional techniques such as questionnaire, interview, and think-aloud remain important for capturing self-reported data, but that there are experimental variables to be "measured, modelled and formalized". It is also the viewpoint expressed in [12], where authors believe that psychophysiological measurements provide important possibilities in product development and can help to deepen and expand the insights gathered by traditional methods. In [13] a method is presented for continuously modelling emotion using physiological data.

In relation with the participation of people with disabilities in affective computing experiences, in [14] a classification based on different stages of affect processing, such as sensing, recognizing, interpreting, selecting, and expressing affects is proposed. Based on that classification, [15] compiles a list of impairments or disorders involving deficiencies in affective processing, as well as the affective computing technologies which are useful in addressing such deficiencies in several application contexts. Visual, hearing and mobility impairments, learning, developmental and anxiety disorders are included. Specifically, for people with visual impairments (including low vision and blindness) using chats or videoconferences, problems in 
sensing visual affective information (face and body gestures) are reported. Furthermore, facial affect recognizer and emotional text readers are proposed as useful affective computing technologies for the aforementioned computer mediated services. According to [16], unaided visually impaired lose 55\% of the affective information that people without those sensorial impairments are able to process. Similarly, in [17] it is referred that "important information in a conversational exchange comes from body language, voice prosody, facial expressions revealing emotional content, and facial displays connected with various aspects of discourse". Furthermore, it is widely agreed that spontaneous facial expressions produced by blind people are the same as those of sighted under the same elicitatory emotional situations. However, in blind individuals occur frequently head and eye blindisms, which do not involve the facial muscles associated with emotions, but rather have more to do with an attempt to improve orientation for maximizing the information coming from audio stimuli $[18,19]$. That has to be taken into account while recoding facial expression in order to assess affective characteristics of the computer interaction of blind people. This seems especially important among connately blind people. Anyhow, whatever gathering methods are used, and according to [20], a multi-method multi-operation measurement approach should be adopted, including triangulation of mixed measures through appropriated protocols.

Furthermore, a number of issues related to conducting UX evaluations with disabled and older users need to be raised [21], such as whether they will use assistive technologies in using the system under evaluation. It is also important that the needs of participants in the evaluation are taken carefully into consideration. Personnel running the evaluations need to be sensitive to their needs. Issues to consider include transport, accessibility of the evaluation facility, accessibility of explanatory materials and consent forms available in the appropriate alternative formats, suitability of the pace of the evaluation for the participants (e.g. older participants may appreciate a slower pace of evaluation).

\section{Experiment at the Madrid Science Week}

To detect emotions from users' interactions in e-learning environments, we designed a series of large-scale experiments for the 2012 Madrid Science Week (MSW) (based on several previous pilot studies) that recorded multiple measures (qualitative, selfreported, physiological and behavioral) during the execution of cognitive tasks. We proposed four activities in the accessible dotLRN learning platform, 1 collaborative and 3 individual. In this paper we are only referring to the individual activity so called "Ambient intelligence: an affective mathematical tutor for daily life mathematics", where 18 people took part. Sessions were scheduled every two hours, with a capacity for up to 4 participants (i.e. the number of experimental stands we had). In each experimental stand there was one researcher (tutor) in charge of recording the measures and supporting the participant along the session. An additional researcher was taking notes of relevant physical movements of all participants. There was also a master guiding the activity in a synchronous way. Several sensors and devices were used to collect data from users' affective states: i) a sensor belt with the following sensors (ECG, GSR and respiratory rate; ii) Kinect for Windows for face features extraction; 
iii) webcam (with integrated microphone); and iv) keyboard and mouse (via a keylogger and a mouse tracker). Along with the physiological and behavioral recordings, participants filled in some personality trait questionnaires as these characteristics relate to how students respond to attempts to provide them affective scaffolding [22]. The data gathered by the sensors was complemented with emotional feedback collected directly from learners in terms of the SAM scale [23] to measure emotions in a dimensional space [24]. The activity was divided into several phases and tasks:

Phase 1 (Preparation) consisted in: 1) Welcome. Participants were thanked for their participation, assigned a stand for the activity and introduced to their tutor; 2) Information Consent. Participants were informed on the activity and asked their permission to gather their participation data anonymously. All participants agreed to have their data recorded; and 3) Sensors attachment. Each tutor was in charge of attaching the physiological sensors to the participants. In order to acquire the electrocardiogram signal, disposable $\mathrm{Ag} / \mathrm{AgCl}$ electrodes were placed in the chest and also bilaterally on the internal side of each ankle. The respiratory rate was recorded by means of Pneumograph belt strapped around the chest. Regarding the GSR another two $8 \mathrm{~mm}$ snap style $\mathrm{Ag} / \mathrm{AgCl}$ pellet embedded into a hook and loop fastener band were attach to the middle phalanx on the index and middle finger of the non-dominant hand (not to interfere with the typing and mouse control). The temperature sensor was attached directly to the internal side of the non-dominant hand wrist by means of a tennis wristband. Participants adopted a normal setting position all through the session and they were instructed not to cross legs.

Phase 2 (Getting Information) consisted in: 1) Demographic information. Data, such as gender, age, studies, occupation, illness related to heart and brain, physical activity, emotional control and technological experience was collected by the tutor; 2) Personality questionnaires. Participants were asked to fill in the Big Five Inventory (BFI) [25] to reveal the main five structural dimensions of personality (Openness, Conscientiousness, Extraversion, Agreeableness and Neuroticism) and the General Self-Efficacy Scale (GSE) [26] to assess the self-beliefs of participants to cope with a variety of difficult demands in life. In the pilot experiments, the questionnaires were available on-line at the platform. However, it was found that filling in printed questionnaires was more convenient as it consumed considerably less time. 3) Data gathering launching. Once participants filled out the questionnaires, sensors were launched (e.g. physiological sensors, webcam and Kinect) as well as the tracking software (i.e., mouse, keyboard and computer tracking).

Phase 3 (Calibration and pre-baseline) consisted in: 1) Physiological sensors baseline. Participants were asked to relax themselves for 2 minutes in order to measure their physiological signals in the absence of any external stimulation; 2) Expectative report. Participants were asked to type short paragraph about their expectations for the activity, both to find out the level of involvement on the upcoming activity and to have some data to compare the typing behavior with the data obtained during the experiment; 3) Polygraph task. With the goal of calibrating elicited emotional response, 7 questions, which fluctuated from neutral (e.g. "Is Paris the capital of France?") to very lock-in ones (e.g. "Have you ever take advantage of a colleagues' work?"), were formulated; 4) Emotional images. 8 images selected from the International Affective Picture System (IAPS) [27] database -rated by a normative samplecovering the emotional dimensional spectrum were shown to the participants in an 
emotional incremental way. Participants were asked to rate valence and arousal with the SAM scale.

Phase 4 (Mathematical activity) consisted in: 1) Problems. Three groups of problems were given to the participants. The first group contained simple mathematical problems to see how each participant reacted when doing mathematical tasks, such as "Arthur has as many euros as the lowest 3-digit number. Adela has as many euros as the biggest 2-digit number. One of them lost one euro and then, both of them had the same amount of euros. Who did lose the euro?". The second one had an equivalent difficulty level that previous. Nevertheless, participants were told that they were even easier than the previous ones, and therefore the time need to accomplish them was limited to 3 minutes. The goal here was to try to elicit certain level of frustration and stress. In the third one simple graphical logical series were presented. The goal here was to try to elicit a relief from previous task and finish the session with a sensation of joy and happiness. The structure was the same for each group of problems. First, a question and four possible answers, with only one correct, were provided. Once participants selected one alternative and submit the response, explained feedback was provided. Then, they had to fill in the SAM scale, similarly as done with the images task; 2) Emotional reports. After each group of problems, participants had to write corresponding paragraphs regarding how they had felt when doing the problems, what they were thinking, what problems did they cope and how did they dealt with them.

Phase 5 (Closing) consisted in 1) Physiological sensors baseline. Participants were asked, again, to relax themselves for 2 minutes in order to check the recovery. Sensors were withdrawn; 2) Feedback from participant. Participants were asked to fill in the Positive and Negative Affect Schedule (PANAS) [28] that consists of 20 adjectives that describe different emotional states; 3) Feedback to participant. Participants were told about the purpose of the tasks they had carried out, debriefed and dismissed.

\section{$4 \quad$ Inclusive Experiment}

The participation of users with disabilities in the MSW experiment implies several challenges. On the one hand, the accessibility and usability aspects of the interaction with the hardware and software used in the experiment needed to be addressed. On the other hand, applicability of computer-based affect detection techniques to users with different abilities needed to be appropriately analyzed. Thus, to make the experiment accessible to students with visual impairments, several adaptations were made. Before going into the details, there are 3 general comments: 1) All the software and the electronic materials prepared for the MWS experiment tasks were chosen/produced with accessibility in mind; 2) Material's accessibility was tested with the same accommodations and assistive technologies (e.g., Jaws screen reader) the participants declared to use; 3) In case any participant declared to use a different version from the one available at the laboratory, relevant differences were briefed before the experiment started. Modifications per phases and tasks are the following:

Phase 1 (Preparation). In the Welcome task, a team member was waiting for the participant at the building entrance upon their arrival by taxi, in order to welcome and guide them to the laboratory. In the Information Consent task, days before the session 
took place an accessible electronic version (MSWord) of the document was emailed to the participants. They could either reply to the invitation email with a statement saying they were willing to participate or print and sign a hard copy of the consent, maybe with the help of a personal assistant. In the Sensors attachment task, special emphasis was made in describing what particular sensor was to be positioned, and how it was to be done.

Phase 2 (Getting Information). In the Demographic information task, additional information was asked: i) level and cause/time of the impairment; ii) accommodations needed to use the computer; and iii) their skills with their assistive technologies (e.g., novice, advanced, etc.). In the Personality questionnaires task, the first participant (a blind person using Jaws screen reader) was told to use the e-learning platform to access the questionnaires on-line. But as previously noticed in the pilots of the MSW, the time required to fill them in was too long. Therefore, for the other two participants, adapted hardcopy versions were done: a Braille version issued by the Spanish National Organization for the Blind (ONCE) and an Arial 14 pt bold version produced by our team.

Phase 3 (Calibration and Pre-baseline). Modifications were done to the Polygraph task. Visual contents from MSW were substituted by an alternative item without visual content. Equivalent emotional elicitation level was used. In the Emotional images task, 8 standardized emotional sounds, ordered from the least to the most unpleasant and from the least to the most arousing, from the International Affective Digital Sounds (IADS) [29] were used to calibrate the emotional response instead of IAPS. Sounds were presented binaurally through headphones. Participants were asked to judge them in valence and arousal dimensions by using a 9-point scale, instead of the SAM, similarly to what was done with the original images.

Phase 4 (Mathematical Activity). Modifications were done to the Problems task. In particular, for the limited time problems, to compensate potential lower browsing speed for either screen reader or enlarged screen users, 1 extra minute was allowed in comparison with the original sessions. Anyhow, and similarly to the original session, allowed time was not enough to fulfill the task completely. It allowed participants to complete the first couple of problems, so that the time could seem realistic to them. The graphical logical series were replaced with alphanumerical or numerical logical series, with similar level of difficulty.

Phase 5 (Closing), the same adaptation as for the personality questionnaires were done in the Feedback from participant task.

\section{Discussion of Results from the Experiments with Participants with Disabilities}

In this section we present some qualitative results from the experiments with the 3 participants with disabilities (two blind and one with low vision). It is noteworthy that with that reduced amount of participants, the analyses that can be performed are partial, taking into account that all of them performed the experiment under different 
access conditions. The purpose of the experience was not to get conclusive results but to bear out the main challenges and difficulties that were involved. The first limitation for a homogeneous gathering of data is the fact that the sources of information were not evenly collected for all the participants. For example, the mouse interaction was only used for one of them (i.e. not totally blind) which performed the task in a very similar manner that the ones on MSW sessions. The visual impairment required the adjustment of font size on screen or paper and the luminosity level, as well as reducing the sight distance form screen -very close distance, approximately $20 \mathrm{~cm}$. This adaptation interfered with the collection of facial expression information, given that the distance from the Kinect and the framing area were out of range most of the time.
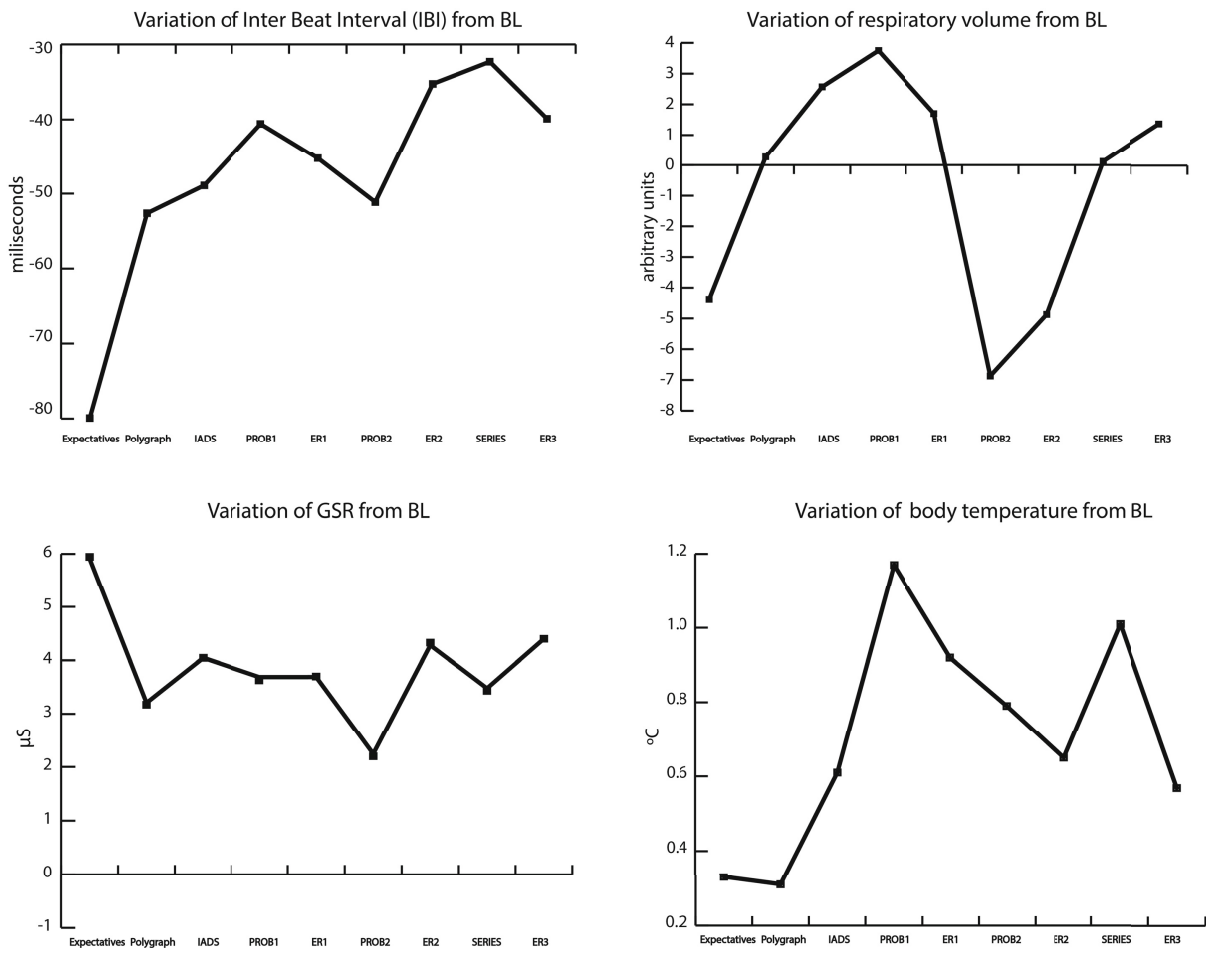

Fig. 1. Evolution of physiological signals through task phases for one of the participants with disabilities respect to baseline (BL)

Another issue to be considered regarding Kinect and totally blind participants (especially when acquired from birth) is the need to use a filter to withdraw the "blindism movements", not associated with emotional reactions that eventually could be present on the recording. Moreover, when participants are blind a discrimination of the keyboard used for browsing by means of Jaws or the typing behavior itself is needed. Keyboard is one of the affective information sources and in this context interferes with the navigation usage. On top of that problem is the increase of finger movements among Jaws users while browsing (increasing the noise on GSR data). 
Regarding the physiological data, using a general descriptive approach, variations related to tasks were observed. Graphics taken from one of the participants are depicted in Fig 1. Reactions towards the tasks were observed, appearing variations among different phases of the experiment. Especially those reactions were observed during the three problem resolutions phases: easy ones, time-limited and alphanumerical series. Data shows a smaller reaction in initial phases as compared with problem phases, and especially with the time-limited one. In the latter, the biggest reaction was observed in all variables, coinciding with users self-reporting stress sensation. Also, emotional sounds generated relevant changes across all the physiological variables. It is also observed a great change in all the emotional reports (ER1, ER2, ER3) filled after the problems (PROB1, PROB2, SERIES3). These changes can be consistent with the fact that participant increase motor demands as this task implied typing.

\section{Conclusions and Ongoing Work}

Inclusive detection of users' affective state while using e-learning resources implies several challenges. The experience described in this paper aimed to detect emotions in an e-learning system by recording multiple measures (qualitative, self-reported, physiological and behavioral) from users, including people with visual impairments.

In order to ensure the inclusiveness of the experiment, a two-stage pilot has been planned and implemented. During the pilot, several issues have been addressed. The accessibility for the target groups regarding all the experience procedures and materials has been ensured, including the tasks, logistics, the informed consent and the recognition certificate. Regarding the materials used for the tasks where emotional responses were measured, which posed accessibility problems for visually impaired people, adapted media (e.g. sound instead of image, alphanumerical or numerical logical series instead of graphical logical series) have been provided. Despite generic accessibility guidelines have been observed, specific adaptations may be needed for users with other impairments (e.g. mobility, hearing impairments, etc.).

Reactions have been identified after the analysis of recorded physiological variables (especially during the execution of limited-time problems, as it is depicted on Fig. 1). However, the remaining recorded variables deserve a careful analysis, to check to what extent they could be considered as a reliable source of emotional information (e.g. disentangle keyboard usage from browsing or typing). They are particularly interesting because they require no devices attached to the participant, increasing the ecological validity of the experiments that aim to study the interaction with e-learning platforms and to elucidate the role of emotions in such an interactions. Additionally, the noise extraction of that signals is a key aspect that should be addressed. Moreover, it would be desirable to carry out a more extensive data collection to be able to perform further analyses and comparisons among all the recorded variables for different access profiles (e.g. Jaws, ZoomText, Braille Refreshable Display, etc.). It would be of great value to know whether the type of access generates an emotional reaction itself (as a consequence of an increase of cognitive load) added to the one elicited by the ongoing task (e.g. additional source of stress). 
As a conclusion it can be stated that elicited emotions by means of the type of tasks manipulation were reflected in the recorded variables for participants with disabilities in an analogous way that happened among the participants of the MSW without disabilities, as expected. Nevertheless, although the adaptations of the recording procedures that were followed seem to have been of worth, some problems arose. In this sense the recording of facial expressions and the analysis of the typing behavior deserve more attention in future works. Finally, additional physiological or neurological measurement (e.g. EEG) could result of help in order to better characterize affective reactions during learning for people with disabilities.

Acknowledgments. Authors would like to thank the participants of the experiments as well as their colleagues of the MAMIPEC project (TIN2011-29221-C03-01), which has been funded by the Spanish Ministry of Economy and Competence.

\section{References}

1. Santos, O.C., Boticario, J.G., Arevalillo-Herraez, M., Saneiro, M., Cabestrero, R., del Campo, E., Manjarres, A., Moreno, P., Quiros, P., Salmeron-Majadas, S.: MAMIPEC Affective Modeling in Inclusive Personalized Educational Scenarios. IEEE Learning Tech. Newsletter 14(4) (October 2012)

2. ETSI: EG 202848 ETSI Guide Human Factors; Inclusive eServices for all: Optimizing the accessibility and the use of upcoming user-interaction technologies. European Telecommunication Standards Institute (2011)

3. Porayska-Pomsta, K., Mavrikis, M., Pain, H.: Diagnosing and acting on student affect: The tutor's perspective. User Modeling and User-Adapted Interaction 18, 125-173 (2008)

4. Carberry, S., de Rosis, F.: Introduction to special issue on 'affective modeling and adaptation'. User Modeling and User-Adapted Interaction 18, 1-9 (2008)

5. Hassenzahl, M., Tractinsky, N.: User experience-a research agenda. Behaviour \& Information Technology 25(2), 91-97 (2006)

6. Tai, M., Woolf, B., Arroyo, I.: Using the Think Aloud Method to Observe Students. In: Help-seeking Behavior in Math Tutoring Software, ICALT 2011 (July 2011)

7. Stathopoulou, I.O., Alepis, E., Tsihrintzis, G.A., Virvou, M.: On assisting a visual-facial affect recognition system with keyboard-stroke pattern information. Knowledge-Based Systems 23, 350-356 (2010)

8. Arroyo, I., Mehranian, H.: A Data Mining Approach to Intelligent Tutoring. Journal of Educational Data Mining, JEDM (2012)

9. Arroyo, I., Cooper, D.G., Burleson, W., Woolf, B.P., Muldner, K., Christopherson, R.: Emotion Sensors Go To School. AIED (2009)

10. Zeng, Z., Pantic, M., Roisman, G.I., Huang, T.S.: A survey of affect recognition methods: audio, visual, and spontaneous expressions. IEEE Transactions on Pattern Analysis And Machine Intelligence 31, 39-58 (2009)

11. Law, E.L.C., Schaik, P.: Editorial: Modelling user experience-An agenda for research and practice. Interacting with Computers 22 22(5), 313-322 (2010)

12. Ganglbauer, E., Schrammel, J., Deutsch, S., Tscheligi, M.: Applying psychophysiological methods for measuring user experience: possibilities, challenges and feasibility. In: User Experience Evaluation Methods in Product Development, UXEM 2009 (2009) 
13. Mandryk, R.L., Atkins, M.S.: A fuzzy physiological approach for continuously modeling emotion during interaction with play technologies. International Journal of HumanComputer Studies 65, 329-347 (2007)

14. Hudlicka, E.: To feel or not to feel: The role of affect in human-computer interaction. International Journal of Human-Computer Studies 59, 1-32 (2003)

15. Garay, N., Cearreta, I., López, J.M., Fajardo, I.: Assistive technology and affective mediation. Human Technology: An Interdisciplinary Journal on Humans in ICT Environments 2, 55-83 (2006)

16. Mehrabian, A.: Silent messages. Wadsworth, Oxford England (1971)

17. Lisetti, C., Nasoz, F.: Using Non-invasive Wearable Computers to Recognize Human Emotions from Physiological Signals. Journal on Applied Signal Processing - Special Issue on Multimedia Human-Computer Interface 11 (2004); EURASIP (ed.)

18. Galati, D., Miceli, R., Sini, B.: Judging and coding facial expression of emotions in congenitally blind children. Int. Journal of Behavioral Development 25, 268-278 (2001)

19. Matsumoto, D., Willingham, B.: Spontaneous facial expressions of emotion of congenitally and noncongenitally blind individuals. Journal of Personality and Social Psychology 96, 1-10 (2009)

20. Gray, W.D., Salzman, M.C.: Damaged merchandise? A review of experiments that compare usability evaluation methods. Human-Computer Interaction 13, 203-261 (1998)

21. Petrie, H., Bevan, N.: The evaluation of accessibility, usability and user experience. The Universal Access Handbook, 10-20 (2009)

22. Robison, J., McQuiggan, S., Lester, J.: Developing Empirically Based Student Personality Profiles for Affective Feedback Models. In: Aleven, V., Kay, J., Mostow, J. (eds.) ITS 2010, Part I. LNCS, vol. 6094, pp. 285-295. Springer, Heidelberg (2010)

23. Bradley, M.M., Lang, P.J.: Measuring emotion: The Self-Assessment Manikin and the semantic differential. J. of Behavior Therapy and Experimental Psychiatry 25, 49-59 (1994)

24. Mehrabian, A.: Pleasure-arousal-dominance: A general framework for describing and measuring individual differences in temperament. Current Psychology: A Journal for Diverse Perspectives on Diverse Psychological Issues 14, 261-292 (1996)

25. John, O.P., Donahue, E.M., Kentle, R.L.: The Big Five Inventory-Versions 4a and 54. University of California, Berkeley, Institute of Personality and Social Research (1991)

26. Schwarcer, R.: Measurement of perceived self-efficacy. Psychometric scales for crosscultural research. Freie Universit., Berlin (1993)

27. Lang, P.J., Bradley, M.M., Cuthbert, B.N.: International affective picture system (IAPS): Affective ratings of pictures and instruction manual. Technical Report A-8. University of Florida, Gainesville, FL (2008)

28. Watson, D., Clark, L.A., Tellegen, A.: Development and validation of brief measures of positive and negative affect: The PANAS scales. Journal of Personality and Social Psychology 54, 1063-1070 (1988)

29. Bradley, M.M., Lang, P.J.: International Affective Digitized Sounds (IADS): Stimuli, instruction manual and affective ratings (Tech. Rep. No. B-2). Gainesville, FL: University of Florida (1999) 